Revista Internacional de Apoyo a la Inclusión, Logopedia, Sociedad y Multiculturalidad.

Volumen 5, Número 2, Junio 2019, ISSN: 2387-0907.DOI: https://dx.doi.org/10.17561/riai.v5.n2.2

\title{
A qualidade de vida e a contribuição da Enfermagem no cuidado ao idoso para promoção à saúde
}

(The quality of life and the contribution of nursing in care to the elderly for health promotion)
Kemle Senhorinha Rocha Tuma
Universidade do Pará- UPA, Belém, Pará (Brasil)
traducion1@gmail.com

Fecha recepción: 11-03-2019

Páginas 14-24

Fecha aceptación: 20-05-2019

\section{Resumo.}

O estudo teve como objetivo analisar a qualidade de vida e a contribuição da enfermagem no cuidado ao idoso, na promoção da saúde e vida saudável. Esta temática é de grande relevância para descrever as formas de melhorias na qualidade de vida. Para tal, foi realizado uma pesquisa de revisão integrativa da literatura. 0 levantamento bibliográfico foi realizado através de pesquisa em artigos publicados entre 2010 a 2017, sendo no total 19 artigos analisados. Os artigos foram coletados nas bases de dados eletrônicos, tais como: Revistas Brasileiras; Biblioteca Virtual de Saúde (BVS); LILACS e SCIELO e outras disponíveis. Os resultados mostram que há a necessidade de incentivar as pessoas idosas a promover melhorias na qualidade de hábitos saudáveis para amenizar a questão das doenças que possam surgir em razão da idade. Conclui-se que a equipe de enfermagem precisa estar envolvida, no sentido mobilizar ações nos diversos aspectos voltados aos determinantes do envelhecimento e possibilitar a valorização e dignidade ao idoso.

Palavras-chave: Idoso; qualidade de vida; cuidados de enfermagem

\begin{abstract}
.
The objective of the study was to analyze the quality of life and the contribution of nursing in the care of the elderly, in the promotion of health and healthy life. This theme is of great relevance to describe ways of improving quality of life. For this, an integrative literature review was carried out. The bibliographic survey was carried out through research on articles published between 2010 and 2017, being in total 19 articles analyzed. The articles were collected in the electronic databases, such as: Brazilian Journals; Virtual Health Library (VHL); LILACS and SCIELO and others available. The results show that there is a need to encourage older people to promote improvements in the quality of healthy habits to alleviate the issue of diseases that may arise due to age. It is concluded that the nursing team needs to be involved, in order to mobilize actions in the various aspects focused on the determinants of aging and to enable the elderly to be valued and dignified.
\end{abstract}

Key Words: elderly; quality of life; nursing 


\section{1.- Introdução.}

Estudos apontam um aumento demográfico de envelhecimento que está ocorrendo de uma maneira acelerada, conforme a Organização Mundial de Saúde (OMS) e 0 Instituto Brasileiro de Geografia e Estatística (IBGE); pois a medida em que 0 envelhecimento populacional aumenta, eleva-se também, a expectativa de vida.

Segundo a OMS, no ano de 2025, será de 73 anos. No Brasil, a expectativa de vida passará de 67 anos para 74 anos, em 2025, ocasionando a duplicação no número de idosos no país que, atualmente, correspondem a mais de 18 milhões de indivíduos com mais de 60 anos de idade. A população está passando por um processo de envelhecimento progressivo, sendo necessária uma maior importância na garantia da qualidade de vida do idoso, de forma adequada para sua sobrevida (Horta \& Ferreira, 2010; p. 34).

Com o envelhecimento saudável, a pessoa idosa almeja uma vida de autonomia, ou seja, ser capaz de decidir e executar suas próprias ações. O que torna uma realidade na existência de um equilíbrio em suas diversas dimensões funcionais; mesmo com suas limitações. Isso não significa a total ausência de problemas nas funções biológicas, pois o ser humano, com idade de 80 anos, mesmo com capacidade de conduzir sua vida, pode ser acometido de agravos e riscos, principalmente de perdas das funções.

Articular ações que promovam a saúde dos idosos, como atividades de rotina e bemestar, é muito importante na condução de uma vida saudável, pois mostra que 0 idoso é capaz de realizar diversas atividades, afazeres e trabalhos, assim como ter convívio social, mesmo com uma idade mais avançada, mas seguramente entendida como uma pessoa saudável em sua capacidade física.

De acordo com Rinaldi (2013), é essencial estabelecer ações e atividades que possam manter a capacidade funcional, principalmente para os profissionais que atuam ou atuarão na atenção à saúde. A independência no cotidiano estabelece 0 equilíbrio da pessoa idosa para uma perspectiva de longevidade na sua forma ativa, exaltando a velhice como uma nova fase da sua história. Envelhecer com saúde possibilita uma vida saudável e feliz do ser humano.

Foram utilizados na pesquisa os seguintes descritores: idoso, qualidade de vida, cuidados de enfermagem.

O interesse surgiu a partir da referência na assistência ao idoso nas suas enfermidades e cuidados, através da atuação do profissional na enfermagem para a elaboração de ações capazes de alcançar a promoção a saúde e qualidade de vida. São necessários os cuidados de enfermagem para auxiliar, promover uma conscientização quanto aos riscos e consequências. A partir destas justificativas emergiu a questão norteadora para esta pesquisa que é: Qual a contribuição da enfermagem no cuidado ao idoso para a promoção da saúde? 
A pesquisa é de grande relevância para a população, especialmente aos idosos e para os profissionais da saúde, com foco na enfermagem. É necessário identificar os problemas quanto ao processo de envelhecer, desenvolver a prevenção, promoção e a proteção do idoso para a melhoria da qualidade de vida e assim, contribuir no exercício da cidadania. Estudos sobre esta temática são fundamentais para se conhecer as verdadeiras demandas com 0 objetivo de apresentar as possibilidades de um envelhecimento saudável, através de ações das políticas públicas e do profissional de enfermagem, como sujeito das ações de saúde.

\section{2.- Processo de envelhecimento.}

O processo de envelhecimento inclui sistemas simbólicos que permeiam a história da sociedade como os aspectos culturais, políticos e econômicos. Tal processo é para a vida toda e estes padrões são formados, de acordo com o princípio da vida que se leva e que pode promover um envelhecimento com saúde ou não.

A velhice em nossa sociedade representa uma fase que atribui muitas perdas, razão pela qual o risco de solidão é eminente e traz muitas consequências que afetam tanto a produção funcional, como a financeira, levando ao aparecimento de muitas patologias que contribuem negativamente na auto estima da pessoa idosa. Nesse período, é necessário que haja compreensão e humanização para 0 enfrentamento desses desafios.

"[...] o envelhecimento não é homogêneo para todos os seres humanos, sendo influenciado pelos processos de discriminação e exclusão associados ao gênero, à etnia, ao racismo, as condições sociais e econômicas, a região geográfica de origem e a localização de moradia." (Rinaldi et all, 2013; p.08).

A valorização e humanização do indivíduo na velhice deve ser respeitada para a uma prestação de assistência igualitária na saúde. Como em todas as etapas da vida do ser humano, o papel da família é muito importante como suporte, fonte de carinho e cuidados, é essencial esse apoio para acolher e oferecer conforto e segurança.

O envelhecimento faz parte da realidade das sociedades no mundo. Pessoas com mais de sessenta anos estão aumentando e a maioria delas vive em países em desenvolvimento. No Brasil, de acordo com o Instituto Brasileiro de Geografia (IBGE) de 2017, estima-se que atualmente existem vinte e seis milhões de pessoas acima de sessenta anos. 


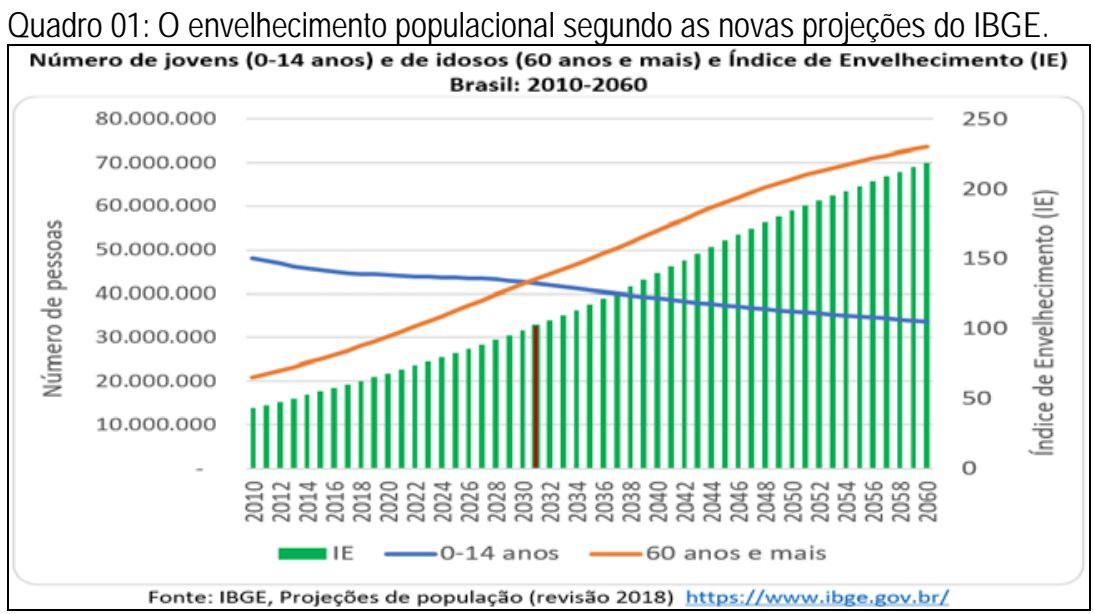

Fonte: $\quad$ https://www.ecodebate.com.br/2018/08/31/0-envelhecimento-populacionalsegundo-as-novas-projecoes-do-ibge-artigo-de-jose-eustaquio-diniz-alves/

$\mathrm{Na}$ medida em que diversos fatores biológicos, sociais, psicológicos, culturais e econômicos influenciam o processo de envelhecimento humano, é importante observar que diferentes aspectos agem na vida do idoso com o objetivo de protegê-lo das situações e condições adversas para a adaptação e superação. Lamond et al. (2009) verificou que o processo de envelhecimento é usualmente caracterizado em termos do estreitamento do círculo de relações significativas, o que faz com que os idosos tenham cerca de metade das relações que tinham no início da vida adulta.

É inevitável, ao envelhecer, as mudanças psicológicas que acontecem no ser humano. Isso quer dizer que o indivíduo, neste processo de envelhecimento, necessita adaptar-se a cada nova situação do seu dia-a-dia. Em relação as modificações sociais, observa-se que as relações se tornam alteradas em consequências da produtividade que diminui, e principalmente, do estado físico e econômico. Esta mudança social é mais evidente em países capitalistas.

\section{1.-Direitos do idoso.}

A Constituição Federal de 1988 expõe em seu artigo 1', inciso III, que 0 Estado democrático de direito tem como fundamento a dignidade da pessoa humana, reconhecendo que todo ser humano tem o direito de ser respeitado como pessoa. Segundo 0 artigo 50, "caput" da Constituição Federal, todas as pessoas devem ser respeitadas, sem nenhuma distinção. Porém, as pessoas idosas, que precisam de uma proteção maior, tem mais dificuldade de se integrar socialmente e não podem ser excluídas da sociedade.

Os Direitos Humanos e Pessoa Idosa é um documento datado em 2005, elaborado e publicado pela Secretaria Especial dos Direitos Humanos, Subsecretaria de 
Promoção e Defesa dos Direitos Humanos da Esplanada dos Ministérios, no qual se discute a concretização dos direitos à promoção de vida e liberdade, assim como, a erradicação das desigualdades e da discriminação contra os idosos. A publicação dos direitos da pessoa idosa é uma contribuição para sensibilização da sociedade aos problemas enfrentados pelos idosos.

De acordo com a Declaração, o direito do idoso está disposto em seu artigo XXV, e diz que toda pessoa tem direito à segurança em caso de doença, invalidez, viuvez $\mathrm{e}$ velhice. Há muitas semelhanças entre os preâmbulos da Declaração Universal dos Direitos Humanos e da Constituição Brasileira de 1988, como por exemplo, a Igualdade e Fraternidade; a Dignidade Humana; a Liberdade; a Justiça e Segurança; a Proteção Legal dos Direitos; a Paz e Solidariedade Universal; o Estado Democrático; os Direitos Sociais e Individuais; e o Bem-estar.

Tanto na Declaração Universal dos Direitos Humanos quanto na Constituição Federal do Brasil, o ideal maior é o de uma sociedade justa e fraterna, pluralista e sem preconceitos de qualquer espécie; de uma sociedade fundada na harmonia social e no compromisso com a solução pacífica de problemas sociais, conflitos e contradições (Oliveira \& Tavares, 2010; p. 15).

\section{2.-A enfermagem e o cuidado ao idoso.}

Dentre os direitos à saúde, assegurados ao idoso, a enfermagem atua dando acesso a informação e aquisição, em relação ao direito do recebimento gratuito de medicamentos, assim como, no cadastramento em base territorial. É atuante também no atendimento em domicílios, nas unidades de saúde, geriátricas e gerontológicas; entre outros recursos direcionados ao tratamento de habilitação ou reabilitação, entre outros.

No que se refere ao profissional de enfermagem e no desempenho de suas atribuições, no contexto legal, este precisa se capacitar para suas atividades ao cuidado do idoso e buscar a melhor forma de assisti-lo, presente também a disseminação de informação da existência deste documento a população idosa e a garantia da existência e do conhecimento de seus direitos. Ou seja, cabe ao enfermeiro, em sua área de atuação, ser um agente intermediador entre a legislação, 0 idoso e a sociedade.

O cuidado ao idoso no domicílio depende do profissional para seu atendimento e a colaboração da família. Nesse contexto, devem ser avaliados o cuidado e o suporte adequado para adotar estratégias de apoio ao idoso. Vale destacar a necessidade de mudanças na forma de atender, no tocante ao objetivo das consultas e das ações de cuidado e orientação. Profissionais especializados na área gerontogeriátrica são fundamentais para essa população (Barreto, 2017; p. 76).

A assistência domiciliar constituirá estratégia importante para diminuir o custo da internação, pois é menos onerosa do que a internação hospitalar. $O$ atendimento ao 
idoso enfermo, residente em instituições, terá as mesmas características da assistência domiciliar. A implantação do hospital dia geriátrico deverá ser estimulada, e terá como objetivo viabilizar a assistência técnica adequada para pacientes cuja necessidade terapêutica e de orientação para cuidadores não justifique a permanência em hospital (Vivian \& Arsimon, 2009; p. 44).

\section{4-O papel do enfermeiro no cuidado ao idoso.}

O planejamento e a implementação do cuidado de enfermagem devem basear-se nas características clínicas identificadas através da observação constante no relacionamento enfermeiro-paciente, que deve ser tranquilo, de forma amigável, compreensivo e sério, visando sempre o aumento da autoestima do paciente. 0 cuidar em enfermagem tem como finalidade prioritária amenizar o sofrimento humano, manter a dignidade e facilitar meios para manejar as crises e as experiências do viver e do morrer, atuando na promoção, recuperação e reabilitação da saúde e qualidade de vida (Oliveira, \& Tavares, 2010; p. 63).

Por isso acredita-se que este estudo contribuirá para o conhecimento e aprendizagem do profissional enfermeiro no atendimento do idoso, contribuirá para a comunidade acadêmica no sentido de mais informação sobre esta área de pesquisa.

0 profissional enfermeiro tem grande responsabilidade no autocuidado com 0 paciente idoso em propor ações que poderão ser realizadas para a minimização ou prevenção de sinais e sintomas para diversas enfermidades. Desta forma evidenciase que as atividades estão proporcionando aos idosos, alegria, participação e convívio tanto familiar quanto com outras pessoas.

O enfermeiro em seu dia a dia deve buscar melhorar o atendimento ao idoso, criando estratégia de implementação de cuidados tanto pelo enfermeiro, quanto pela equipe, contudo faz-se necessário desenvolver uma visão sistêmica e integral do idoso, família e comunidade que assuma na prática a inclusão de ações de saúde, contribuindo um real avanço na construção e reorientação do processo de trabalho na atenção básica, atuando com criatividade, mediante uma prática humanizada e competente envolvendo ações de prevenções para reabilitação dos indivíduos envolvidos no seu processo de cuidar (Abreu, 2013; p. 85).

Para Abreu (2013) evidencia-se a necessidade de inserir efetivamente as ações de prevenção ao adoecimento nas unidades de saúde da família para que se possa desenvolver um cuidado integral ao usuário idoso a família e comunidade. Cabe ao profissional enfermeiro ter habilidades para saber identificar sinais e sintomas o mais precocemente possível, estabelecer ações de enfermagem; acompanhar 0 tratamento do idoso, ajudando-o na sua recuperação.

O enfermeiro é responsável pela estruturação do conhecimento das respostas humanas aos problemas de saúde, tem o intuito de propiciar ao indivíduo as melhores condições para descrever e desenvolver um plano de cuidados 
fundamentado cientificamente, e com confiança utiliza-se da identificação dos diagnósticos de enfermagem. Estabelecer uma relação de ajuda entre o enfermeiro e a pessoa idosa no âmbito domiciliar, fundamentada na teoria das relações interpessoais, identificando diagnóstico de enfermagem e suas intervenções correlatada.

Segundo Janine \& Bessler (2015), as intervenções que o enfermeiro deve implementar no cuidado com idoso, de incentivar o mesmo a expressar seus sentimentos especialmente à maneira como se sente ou pensa ou vê de si mesma. Estimulá-lo a fazer perguntas sobre os problemas de saúde, o tratamento e progresso, proporcionar informações confiáveis e reforçar as já conhecidas.

Esclarecer qualquer concepção errônea que a pessoa tenha sobre si mesma, os cuidados e os cuidadores, preparar as pessoas próximas para as mudanças físicas e emocionais, apoiar a família à medida que se adapta, estimulando as visitas dos amigos e das pessoas mais próximas, recomendando que compartilhem com a pessoa seu valor e a sua importância para eles.

As atribuições do enfermeiro no que se refere ao cuidado com o idoso, são: a) Realizar atenção integral às pessoas idosas; b) Realizar assistência domiciliar, quando necessário; c) Realizar consulta de enfermagem, incluindo a avaliação multidimensional rápida e instrumentos complementares, se necessário, solicitar exames complementares e prescrever medicações, conforme protocolos ou outras normativas técnicas estabelecidas pelo gestor municipal, observadas as disposições legais da profissão; d) Supervisionar e coordenar o trabalho dos ACS e da equipe de enfermagem; e) Realizar atividades de educação permanente e interdisciplinar junto aos demais profissionais da equipe; f) Orientar ao idoso, aos familiares e/ou cuidador sobre a correta utilização dos medicamentos.

\section{3.-Metodologia.}

A metodología utilizada na pesquisa apresenta o tipo de pesquisa bibliográfica. Neste sentido, a metodología da pesquisa científica se refere ao camino adotado no desenvolver da pesquisa e as técnicas utilizadas na coleta de dados. Por tanto, segundo Gerhardt \& Silveira (2009) a metodología comprende "el conjunto de conocimientos precisos y metódicamente ordenados en relación a determinado dominio del saber".

Foi realizada uma busca de material bibliográfico com o objetivo de fundamentar a pesquisa. A busca foi estrita de acordo com os seguintes descritores: analisar a qualidade de vida e a contribuição da enfermagem no cuidado ao idoso, na promoção da saúde e vida saudável. Esta temática é de grande relevância para descrever as formas de melhorias na qualidade de vida. Para tal, foi realizado uma pesquisa de revisão integrativa da literatura. 
O levantamento bibliográfico foi realizado através de pesquisa em artigos publicados entre 2010 a 2017, sendo no total 19 artigos analisados. Os artigos foram coletados nas bases de dados eletrônicos, tais como: Revistas Brasileiras; Biblioteca Virtual de Saúde (BVS); LILACS e SCIELO e outras disponíveis

Os autores enumerados na pesquisa auxilian a discussão das ideias, das leyes, do contexto que la enseñanza de la lengua española está inserta y de la comprensión de la necesidad de una educación que contenga más conocimientos y posibilidades para que el alumno se desarrolle en el ámbito personal y profesional con una cualificación adecuada para actuar en el mercado de trabajo.

\section{4.-Resultados e Discussão.}

Quadro 02: Artigos analisados e identificados conforme o quadro.

\begin{tabular}{|l|l|l|l|}
\hline \multicolumn{1}{|c|}{ Fonte } & \multicolumn{1}{|c|}{ Autor } & Ano & \multicolumn{1}{|c|}{ Objetivo do artigo } \\
\hline $\begin{array}{l}\text { 1-SciELO } \\
\text { Brasil }\end{array}$ & $\begin{array}{l}\text { Vivian, A. S.; } \\
\text { ARGIMON, I. I. L. }\end{array}$ & 2009. & $\begin{array}{l}\text { Avaliar os níveis das doenças que } \\
\text { impedem o desempenho dos idosos. }\end{array}$ \\
\hline $\begin{array}{l}\text { 2-SciELO } \\
\text { Brasil }\end{array}$ & $\begin{array}{l}\text { Horta, A. L. M.; } \\
\text { Ferreira, D. C. O.; } \\
\text { Zhao, L. M. }\end{array}$ & 2010 & $\begin{array}{l}\text { Avaliar o enfrentamento do idoso e seus } \\
\text { familiares na atenção } \\
\text { a saúde pública. }\end{array}$ \\
\hline $\begin{array}{l}\text { 3-SciELO } \\
\text { Brasil }\end{array}$ & $\begin{array}{l}\text { Oliveira, J.; Tavares, } \\
\text { D. M. }\end{array}$ & 2010 & $\begin{array}{l}\text { Conhecer a atuação do enfermeiro na } \\
\text { atenção ao idoso. }\end{array}$ \\
\hline $\begin{array}{l}\text { 4-SciELO } \\
\text { Brasil }\end{array}$ & $\begin{array}{l}\text { Farias, R. G.; Santos, } \\
\text { S. M. A. }\end{array}$ & 2012. & $\begin{array}{l}\text { Identificar os fatores determinantes } \\
\text { sobre o envelhecimento ativo. }\end{array}$ \\
\hline $\begin{array}{l}\text { 5-SciELO } \\
\text { Brasil }\end{array}$ & Rinaldi, F.C. et AL. & 2013. & $\begin{array}{l}\text { Conhecer a contribuição para a } \\
\text { promoção do envelhecimento saudável. }\end{array}$ \\
\hline 6-BDENF & Abreu, V. C. et al. & 2013 & $\begin{array}{l}\text { Cuidados com o idoso no programa } \\
\text { saúde da família }\end{array}$ \\
\hline $\begin{array}{l}\text { 7-SciELO } \\
\text { Brasil }\end{array}$ & Cordeiro, J. et al. & 2014 & $\begin{array}{l}\text { Analisar a memória declarativa e } \\
\text { qualidade de vida de idosos. }\end{array}$ \\
\hline 8-BDENF & $\begin{array}{l}\text { Janine, J. P; Bessler, } \\
\text { D; Vargas, A B. }\end{array}$ & 2015 & $\begin{array}{l}\text { Analisar os impactos das ações de } \\
\text { promoção e educação em saúde }\end{array}$ \\
\hline 9-BDENF & Barreto, J. & 2017 & $\begin{array}{l}\text { Analisar as carências sociais no cuidado } \\
\text { ao idoso }\end{array}$ \\
\hline
\end{tabular}

Fonte: Dados da pesquisa, a partir da revisão integrativa da literatura.

A longevidade na visão de Reinaldo (2013) é adquirida por meio de melhor qualidade de vida da população e tem obrigado o setor saúde a enfrentar alguns desafios para proporcionar este bem estar ao idoso, o que tem levado as equipes de enfermagem 
das unidades básicas a não conseguir realizar uma assistência de modo adequada, uma vez que os profissionais se prendem apenas aos aspectos biológicos do envelhecimento, esquecendo que a população da terceira idade também possui uma vida social e afetiva.

A importância da assistência ao idoso é norteada por Abreu (2013), pelos princípios do Programa Saúde da Família para o cuidado integral, a vivência com profissionais da saúde, particularmente com enfermeiros, membros de equipes de saúde da família, permite constatar a presença de limitações no processo de cuidar da pessoa idosa no âmbito desse Programa.

O papel da enfermagem segundo Oliveira e Tavares (2010) é desenvolver práticas, 0 relacionamento interpessoal com cliente e familiares e o cuidado baseado na cientificidade. A ESF constitui-se em espaço privilegiado para atenção integral à saúde do idoso, pois sua proximidade com a comunidade e a atenção domiciliária possibilita atuar de forma contextualizada na realidade vivenciada pelo idoso no seio familiar.

A efetiva inserção do idoso em Unidades de Saúde, sobretudo aquelas sob a ESF, pode representar para ele o vínculo com o sistema de saúde. Considerando as especificidades do processo de envelhecimento e a necessária adequação e qualificação profissional, formação das ações dos enfermeiros na atenção à saúde da população idosa.

Para Rinaldi (2013), que discutem as práticas de assistência, políticas, estratégias e ações governamentais formalizadas para a saúde do idoso, a "humanização" deve se fazer presente em várias discussões, inclusive, tornou-se diretriz da Política Nacional de Humanização, esses pacientes enfrentam, ainda, vários obstáculos para assegurar alguma assistência à saúde. Está em evidência um novo modelo assistencial das estratégias de atenção e gestão no SUS e de formação dos profissionais de saúde. Assim, muito se tem discutido a respeito da humanização na assistência à saúde, provavelmente devido à sua fundamental relevância, uma vez que é baseada em princípios como a equidade, integralidade da assistência, dentre outros, resgatando, assim, a valorização da dignidade do usuário e também do trabalhador.

Horta e Ferreira (2010) discutem a influência do movimento de humanização, a integralidade assistencial possa ser desenvolvida, não apenas como superação de dicotomias técnicas entre preventivo e curativo, entre ações individuais e coletivas, mas como valorização e priorização da responsabilidade pela pessoa, do zelo e da dedicação profissional por alguém, como outra forma de superar os lados dessas dicotomias. Isto é, a humanização induz a pensar que não é possível equacionar a questão da integralidade sem valorizar. 
A longevidade de acordo com Cordeiro (2014), apresenta-se como um fenômeno desafiador nos dias de hoje. Esses desafios são amplos e diversos, exigindo uma atualização da compreensão sobre o processo de envelhecer.

Barreto (2017); Paul (2016) abordam a questão da qualidade de vida dos idosos e a questão das carências sociais no cuidado, a prevenção, a vulnerabilidade, os papeis sociais e a expectativa da sociedade na tomada de decisões e participação do indivíduo no campo de forças.

\section{5.-Conclusão.}

Para se alcançar nível de contentamento perante a vida, precisa ter expectativas do futuro, estabelecer propósitos, ser persistente em algumas ações de melhoria. A partir do momento que uma pessoa com 60 anos ou mais realiza de maneira positiva suas condições de vida, pode-se dizer que elas possuem perspectivas favoráveis.

Os resultados mostram que há a necessidade de incentivar as pessoas idosas e seus hábitos saudáveis para amenizar a questão das doenças que possam surgir em razão da idade e também promover condições favoráveis para sua autonomia. Importante falar do respeito e valorização ao conhecimento adquirido ao longo de sua trajetória de vida. Pois a saúde de uma pessoa deve ser entendida de maneira concreta e ampla, de modo a apresentar resultados de promoção e proteção à saúde decorrentes de trabalhos diversos e que envolvam vários profissionais de saúde que atuem de forma integrada para a promoção à saúde.

Conclui-se que a equipe de enfermagem precisa se envolver, no sentido de mobilizar ações nos diversos aspectos voltados aos determinantes do envelhecimento. Isso merece mais atenção por parte local dos órgãos responsáveis, para assim trabalhar e programar suas ações com foco nas políticas públicas existentes conforme a realidade da região. Finalmente, acredita-se que as mudanças, possam ocorrer com 0 avanço social, de modo a compreender o idoso, na percepção de igualdade e universalidade, onde a sociedade adote políticas mais justas.

\section{6.-Referências.}

Abreu, V. C. et al. (2013). 0 trabalho da enfermagem no cuidado com 0 idoso no programa saúde família: Revisão bibliográfica. Revista formar, interdisciplinar, Sobral. V.1, n. 3, p.23-24, Jul-dez 2013.

Cordeiro, J.; Del Castillo, B. L.; Freitas, C. S. (2014). Gonçalves Marisa Pereira. Efeitos da atividade física na memória declarativa, capacidade funcional e qualidade de vida em idosos. Rev Bras Geriatr Gerontol. 2014;17(3):541-52. https://doi. org/10.1590/1809-9823.2014.13006 
Farias, R.G., Santos, S.M.A (2012). Influência dos determinantes do envelhecimento ativo entre idosos mais idosos. Revista Texto contexto Enfermagem, v. 21 no.1 Florianópolis Jan./Mar.

Gerhardt, T.E., Silveira, D.T.. (2009). Métodos de pesquisa. Porto Alegre: Universidade Federal do Rio Grande do Sul, Editora da UFRGS.

Horta, A. L. M.; Ferreira, D. C. O.; Zhao, L. M. (2010). Envelhecimento, estratégias de enfrentamento do idoso e repercussões na família. Revista Brasileira de Enfermagem. Vol. 63 nº4 Brasília Jul-Agost. 2010.

Janine, J.P., Bessler, D., Vargas, A.B. (2015). Educação em saúde e promoção da saúde: impacto na qualidade de vida do idoso. Rio de janeiro, v.39, 2015.

Oliveira, J., Tavares, D.M. (2010). Atenção ao idoso na estratégia saúde da família: atuação do enfermeiro. Revista da escola de Enfermagem da USP, 2010. Disponível em: < http://www.scielo.br/pdf/reeusp/v44n3/32.pdf>. Acesso em: 03 mai. 2018.

Rinaldi, F.C. et al. (2013). O papel da enfermagem e sua contribuição para a promoção do envelhecimento saudável e ativo. Revista Eletrônica Gestão \& Saúde Vol.04, №. 02, Ano 2013 p.454-66. Disponível em: Acesso em: 03 mai 2018.

Vivian, A.S., Argimon, I.I.L. (2009). Estratégias de enfrentamento, dificuldades funcionais e fatores associados em idosos institucionalizados. Cad. Saúde Pública, Rio de Janeiro, 25 (2): 436 - 444, Fev. 